\title{
Egg consumption and insulin metabolism in the Insulin Resistance Atherosclerosis Study (IRAS)
}

\author{
Chee-Tin Christine Lee ${ }^{1}$, Angela D Liese ${ }^{2}$, Carlos Lorenzo ${ }^{3}$, Lynne E Wagenknecht ${ }^{4}$, \\ Steven $\mathrm{M} \mathrm{Haffner}^{5}$, Marian J Rewers ${ }^{6}$ and Anthony J Hanley $1,7,8, *$ \\ 'Department of Nutritional Sciences, University of Toronto, FitzGerald Building, 150 College Street Room 341, \\ Toronto, ON M5S 3E2, Canada: ${ }^{2}$ Department of Epidemiology and Biostatistics, University of South Carolina, \\ Columbia, SC, USA: ${ }^{3}$ Division of Clinical Epidemiology, University of Texas Health Science Centre, San Antonio, \\ TX, USA: ${ }^{4}$ Division of Public Health Sciences, Wake Forest School of Medicine, Winston-Salem, NC, USA: \\ ${ }^{5}$ Department of Medicine, Baylor College of Medicine, Houston, TX, USA: ${ }^{6}$ Barbara Davis Center for Diabetes, \\ University of Colorado School of Medicine, Aurora, CO, USA: ${ }^{7}$ Department of Medicine and Dalla Lana School \\ of Public Health, University of Toronto, Toronto, ON, Canada: ${ }^{8}$ Leadership Sinai Centre for Diabetes, Mount \\ Sinai Hospital, Toronto, ON, Canada
}

Submitted 20 December 2012: Final revision received 7 May 2013: Accepted 9 May 2013: First published online 19 June 2013

\begin{abstract}
Objective: To examine the association between egg consumption and measures of insulin sensitivity $\left(\mathrm{S}_{\mathrm{I}}\right)$, acute insulin response (AIR) and metabolic clearance rate of insulin (MCRI).

Design: Cross-sectional analysis.

Settings: Egg consumption, categorized as $<1$ /week, 1 to $<3$ /week, 3 to $<5$ /week and $\geq 5 /$ week, was measured using a validated FFQ. $S_{I}$, AIR and MCRI were determined from frequently sampled intravenous glucose tolerance tests.

Subjects: Non-diabetic participants ( $n$ 949) in the Insulin Resistance Atherosclerosis Study (IRAS).

Results: Egg consumption was inversely associated with $\mathrm{S}_{\mathrm{I}}$ and MCRI, and positively associated with fasting insulin in regression models adjusted for demographic, socio-economic, lifestyle and dietary factors $(\beta=-0 \cdot 22,95 \% \mathrm{CI}$ $-0 \cdot 38,-0 \cdot 045, P=0 \cdot 05$ for $\mathrm{S}_{\mathrm{I}} ; \beta=-0 \cdot 20,95 \% \mathrm{CI}-0 \cdot 34,-0 \cdot 055, P=0 \cdot 005$ for MCRI; $\beta=0 \cdot 35,95 \%$ CI $0 \cdot 15,0 \cdot 54, P=0 \cdot 002$ for fasting insulin; all $P$ values for linear trend). These associations remained significant after additionally adjusting for energy intake or dietary saturated fat, although dietary cholesterol and BMI attenuated these associations to non-significance. Egg consumption was not associated with AIR.

Conclusions: Dietary cholesterol and BMI appear to mediate the inverse association of egg consumption with insulin sensitivity and clearance. Alternatively, egg consumption may be clustered with other dietary behaviours which increase BMI, hence negatively impacting on insulin sensitivity and clearance.
\end{abstract}

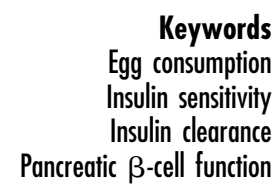

Keywords gensumption Insulin sensitivity Pancreatic $\beta$-cell function
Frequent egg consumption has been linked to increased risk of CVD and mortality in diabetes ${ }^{(1,2)}$. Recent observational evidence has also suggested that eating eggs regularly is associated with a higher risk of developing type 2 diabetes $^{(3-5)}$ and gestational diabetes ${ }^{(6)}$. However, this association has not been consistently demonstrated across different populations. For example, egg consumption was not associated with incident diabetes in older individuals with limited egg intake ${ }^{(7)}$. To date, little is known regarding the potential mechanisms that may explain these reported associations. Since egg consumption is common, it is important to explore how this modifiable exposure is associated with the metabolic traits underlying type 2 diabetes. To our knowledge, only one previous study has investigated the association of egg consumption with insulin sensitivity ${ }^{(7)}$. In a crosssectional analysis of the Cardiovascular Health Study, higher egg consumption was significantly associated with higher fasting blood glucose, fasting insulin and homeostasis model assessment-insulin resistance ${ }^{(7)}$. The association of egg consumption with $\beta$-cell function or insulin clearance has not been examined.

Because of the inconsistent associations of egg consumption with incident diabetes and the limited available evidence on potential mechanisms implicating eggs in diabetes pathogenesis, we aimed to examine the association between egg consumption and detailed measures of insulin sensitivity $\left(\mathrm{S}_{\mathrm{I}}\right)$, acute insulin response (AIR) and 
metabolic clearance rate of insulin (MCRI) from a frequently sampled intravenous glucose tolerance test (FSIGTT) in the multi-ethnic cohort of the Insulin Resistance Atherosclerosis Study (IRAS).

\section{Participants and methods}

\section{Study population}

The present cross-sectional study used data from the IRAS, which recruited 1625 participants from four clinical centres located in San Antonio, TX, San Luis Valley, CO, Oakland, CA and Los Angeles, CA between October 1992 and April 1994. The study design and research methods have been published in detail ${ }^{(8)}$. The institutional review boards of the clinical centres approved the study protocol and all participants provided written informed consent. The sample size of the present analysis was 949 participants, after excluding those with prevalent diabetes at baseline ( $n$ 537), as well as those with missing values of egg consumption, $\mathrm{S}_{\mathrm{I}}$, AIR or MCRI ( $n$ 139).

\section{Data collection}

Participants attended two visits at the baseline examination and again at 5-year follow-up examination. The two visits at each examination occurred approximately one week apart. They were asked to fast for $12 \mathrm{~h}$, to abstain from alcohol and heavy exercise for $24 \mathrm{~h}$ before each visit, as well as to abstain from smoking on the morning of the examination. Height and weight were measured to the nearest $0.5 \mathrm{~cm}$ and $0.1 \mathrm{~kg}$, respectively. BMI was calculated as weight in kilograms divided by the square of height in metres. Waist and hip circumferences were measured to the nearest $0.5 \mathrm{~cm}$ using a steel tape. All measurements were taken in duplicate following standardized procedures and we used the averages of these measurements in the analyses. Blood pressure was measured using a standard mercury sphygmomanometer after participants were rested for $5 \mathrm{~min}$. We used the average of the second and the third readings for analysis. Participants reported demographic and socio-economic information (e.g. age, sex, ethnicity, education, income) as well as lifestyle factors (e.g. smoking, alcohol consumption, physical activity) in standardized questionnaires $^{(8)}$.

\section{Measurement of egg consumption}

At baseline, centrally trained interviewers conducted interviews to complete a semi-quantitative FFQ. The FFQ was designed to assess usual dietary intake in the past year. The 114-item FFQ was adapted from the National Cancer Institute Health Habits and History Questionnaire (NCI-HHHQ) and the food items were expanded to reflect the dietary intake of the diverse IRAS populations. The FFQ was validated against eight $24 \mathrm{~h}$ dietary recalls. The IRAS investigators used the HHHQ-DIETSYS analysis software (version $3 \cdot 0,1993$ ) to analyse nutrient intake from the $\mathrm{FFQ}^{(9)}$. Each item of the FFQ provided nine frequency options, from 'never or less than once a month' to 'six or more times per day'. Participants were asked to indicate how often they consumed each item and to specify whether they consumed a small, medium or large portion compared with individuals in similar age and sex groups. To calculate the intake of each item, the frequency of intake was weighted by the portion size indicated, using a factor of $0.5,1$ and 1.5 for small, medium and large, respectively. The FFQ included one question regarding egg consumption, including omelettes and frittata. We divided egg consumption into four categories: less than one medium egg per week, one to less than three medium eggs per week, three to less than five medium eggs per week and five or more medium eggs per week.

\section{Measurement of insulin sensitivity, $\beta$-cell function and insulin clearance}

A modified FSIGTT was used to measure insulin sensitivity, $\beta$-cell function and insulin clearance ${ }^{(10)}$. Modifications to the original protocol included: (i) insulin, instead of tolbutamide, to ensure adequate levels of plasma insulin to calculate insulin sensitivity accurately across a broad range of glucose tolerance ${ }^{(11)}$; and (ii) a reduced sampling protocol, using twelve instead of thirty samples, because of the large number of participants ${ }^{(12)}$. Insulin resistance, expressed as $S_{I}$, was calculated using minimal model analysis (MINMOD version $3 \cdot 0)^{(13)}$. A higher value of $S_{I}$ indicates an increase in insulin sensitivity. $\beta$-Cell function was measured by AIR defined as the average increase in plasma insulin at time points 2 and 4 min after infusing glucose ${ }^{(14)}$. A higher value of AIR indicates an increase in insulin secretion. MCRI was calculated as the ratio of the insulin dose over the incremental area under the curve of insulin from 20 min to infinity ${ }^{(15)}$ using the following equation:

$$
\operatorname{MCRI}(1 / \mathrm{min})=\frac{\text { Dose } \times 1000}{\int_{t=20}^{\infty}(\operatorname{Ins}(t)-\operatorname{Ins}(0))},
$$

where Dose represents the amount of insulin injected at $20 \mathrm{~min}$, Ins $(t)$ the plasma insulin concentration in standard units at each FSIGTT sampling point and Ins(0) the fasting plasma insulin concentration determined before injecting glucose in the FSIGTT. A higher value of MCRI indicates an increase in insulin clearance.

\section{Biochemical analysis}

Plasma glucose was measured using the glucose oxidase technique on an auto-analyser. Plasma insulin level was determined with the dextran-charcoal RIA ${ }^{(8)}$. Measurements of plasma lipids and lipoproteins were determined at the central IRAS laboratory using the Lipid Research Clinics methods ${ }^{(16)}$. 


\section{Statistical analysis}

We described the characteristics of participants at baseline, stratified by categories of egg consumption, using means and standard deviations for normally distributed continuous variables, medians and interquartile ranges for skewed continuous variables and percentages for categorical variables. We used ANOVA, Kruskal-Wallis tests and $\chi^{2}$ tests to determine whether continuous and categorical variables differed across the distribution of egg consumption. We described the association of egg consumption with potential mediators (e.g. dietary cholesterol, dietary saturated fat, energy intake and BMI) and food choices which may cluster with egg consumption using Spearman's rank correlation coefficient.

We modelled measures of insulin sensitivity, $\beta$-cell function and insulin clearance, including $\mathrm{S}_{\mathrm{I}}$, fasting insulin, AIR and MCRI, as continuous outcome variables. The distributions of these outcome variables were skewed; therefore, we natural log-transformed them for normality. For $\mathrm{S}_{\mathrm{I}}$, we added a constant of one to all values before the log-transformation because of the presence of zero values in the data. We used unadjusted and multivariable-adjusted linear regression to explore the association between egg consumption and each outcome measure. We reported the regression coefficients and their $95 \%$ confidence intervals in each model, and presented the $P$ value for linear trend across categories of egg consumption.

We included covariates in multivariable models if they were risk factors for diabetes based on previous literature, if they were significantly correlated with egg consumption in the IRAS data or if they were of a priori clinical relevance. Potential confounders included age, sex, ethnicity, education, income, smoking, alcohol consumption, energy expenditure, family history of diabetes, and consumption of processed meats, fast foods, red meats, dairy products, vegetables, whole grains, coffee and tea.

We used a staged approach to enter covariates into the regression model. We first adjusted for demographics (i.e. age, sex, ethnicity, study centre), followed by socioeconomic status (i.e. education, income), lifestyle factors (i.e. smoking, alcohol consumption, energy expenditure), family history of diabetes and food items (processed meats, fast foods, red meats, dairy products, vegetables, whole grains, coffee and tea). In this multivariableadjusted model, we then entered dietary factors (i.e. energy, saturated fat and cholesterol), BMI and waist:hip ratio (WHR) one variable at a time to determine if any of these variables had an impact on the associations observed. We used Sobel-Goodman tests, which bootstrap the standard errors of the mediated effects, to determine whether dietary cholesterol and BMI mediated the association of egg consumption with insulin sensitivity and clearance ${ }^{(17)}$. Regression models were based on 949 participants with data on egg consumption $S_{\text {I }}$, AIR and MCRI; sample sizes in full multivariable-adjusted models ranged from 937 to 942 due to occasional missing values on covariates. We examined the interaction between egg consumption and age, sex, ethnicity or BMI status on all outcome variables. Statistical analyses were performed using the statistical software package STATA $12 \cdot 0$.

\section{Results}

Characteristics of the study population, stratified by categories of egg consumption, are shown in Table 1. The median age of the participants was 54 years (range 40-69 years) and 45\% were men. Among these participants, $40 \%$ were Caucasians, $26 \%$ were African Americans and $34 \%$ were Hispanics. Approximately $50 \%$ of the study population reported consuming less than one egg per week. More Hispanics reported eating five or more eggs per week. Participants with higher income and higher educational level reported eating eggs less frequently. Participants who ate eggs regularly were more likely to be male and to smoke. In addition, they had higher values of BMI and WHR, as well as higher dietary intakes of energy, saturated fat and cholesterol. Frequent consumption of eggs was associated with lower values of $\mathrm{S}_{\mathrm{I}}$ and MCRI and higher values of AIR and fasting insulin. There was no significant difference in fasting blood glucose and 2-h post-load blood glucose related to the number of eggs consumed per week. Egg consumption was positively and significantly correlated with dietary cholesterol, dietary saturated fat, energy intake, BMI and WHR, as well as consumption of processed meats, fast foods, red meats, dairy products, vegetables, whole grains, coffee and tea (Table 2).

Egg consumption was inversely associated with $\mathrm{S}_{\mathrm{I}}$ and MCRI, and positively associated with fasting insulin and 2-h post-load blood glucose, in unadjusted regression models and in models adjusted for potential confounders, including demographic, socio-economic, lifestyle and dietary factors and family history of diabetes. Egg consumption was not associated with $\mathrm{S}_{\mathrm{I}}$-adjusted AIR and fasting blood glucose in unadjusted and multivariableadjusted regression models. There was no interaction between egg consumption and age, sex, ethnicity and BMI status on any of the outcomes (Table 3).

In a series of regression models to explore the potential mediating/confounding effects, we observed that the associations of egg consumption with $\mathrm{S}_{\mathrm{I}}$, fasting insulin, MCRI and 2-h post-load blood glucose remained statistically significant after adjusting for energy intake, dietary saturated fat or WHR. However, all of these associations were attenuated to non-significance by dietary cholesterol and BMI (Table 4). The Sobel-Goodman mediation tests showed that dietary cholesterol and BMI are intermediates in the association of egg consumption with insulin sensitivity and clearance (Table 5). 
Table 1 Baseline characteristics of non-diabetic participants ( $n$ 949) in the Insulin Resistance Atherosclerosis Study (IRAS) by distribution of egg consumption

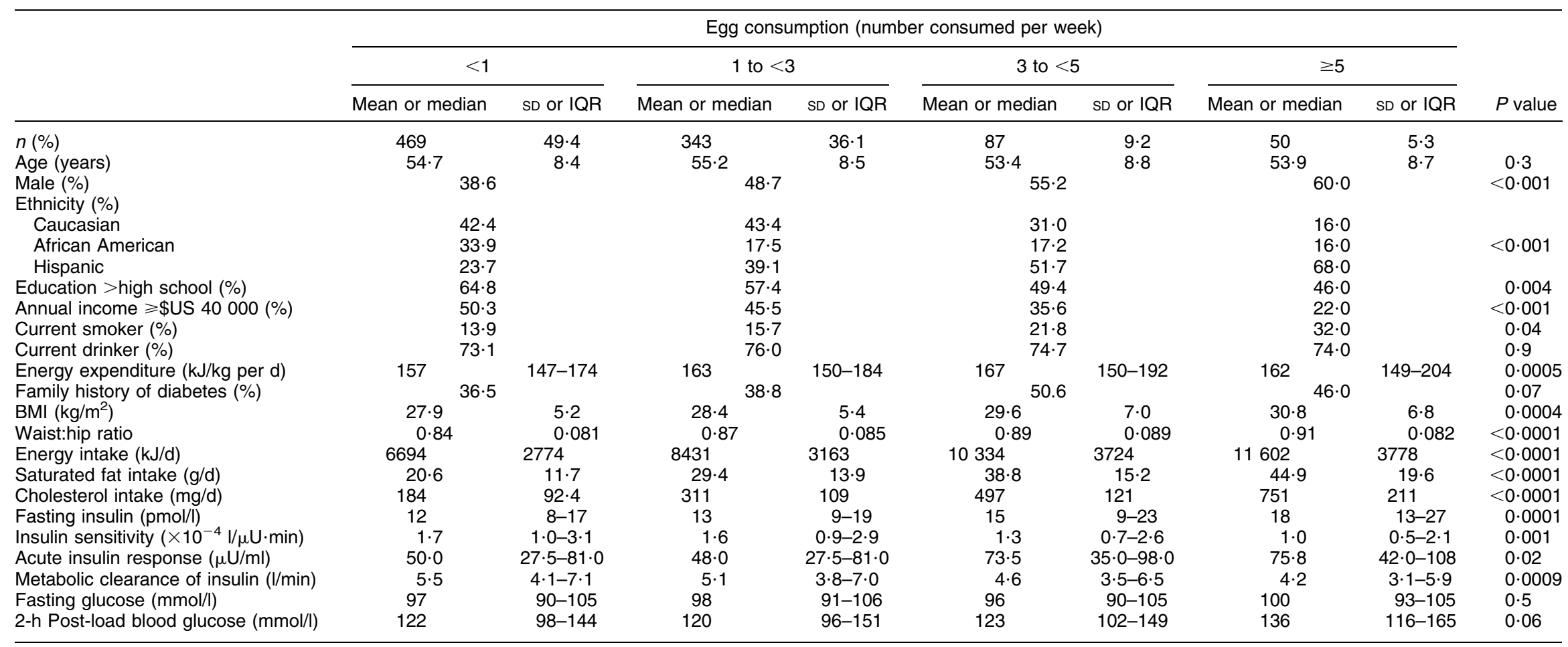

Data are presented as mean and standard deviation, percentage, or median and interquartile range (IQR).

ANOVA, Kruskal-Wallis tests and $\chi^{2}$ tests were used to determine whether continuous and categorical variables differed across the distribution of egg consumption. 
Table 2 Spearman correlation analysis between egg consumption and indices of obesity and food items among non-diabetic participants ( $n$ 949) in the Insulin Resistance Atherosclerosis Study (IRAS)

\begin{tabular}{lcc}
\hline & \multicolumn{2}{c}{ Egg consumption } \\
\cline { 2 - 3 } & $\rho$ & $P$ value \\
\hline Dietary cholesterol & 0.75 & $<0.0001$ \\
Dietary saturated fat & 0.48 & $<0.0001$ \\
Energy intake & 0.43 & $<0.0001$ \\
BMI & 0.10 & 0.002 \\
Waist:hip ratio & 0.18 & $<0.0001$ \\
Processed meats & 0.50 & $<0.0001$ \\
Fast foodst,f & 0.33 & $<0.0001$ \\
Red meats & 0.47 & $<0.0001$ \\
Dairy products & 0.23 & $<0.0001$ \\
Fish & 0.03 & 0.4 \\
Fruits & 0.02 & 0.6 \\
Vegetables & 0.10 & 0.003 \\
Whole grains & 0.15 & $<0.0001$ \\
Coffee \& tea & 0.14 & $<0.0001$ \\
\hline
\end{tabular}

Spearman's rank correlation coefficient was used to describe the correlation of egg consumption with indices of obesity and food items.

*Processed meats refer to ham, sausage, hot dogs, bacon and bologna.

tFast foods refer to burgers, French fries, fried chicken, fried fish and pizza.

fDairy products refer to cheese, milk and yoghurt.

\section{Discussion}

The present cross-sectional study extends the scientific literature on the association of egg consumption with the risk of diabetes. Specifically, while egg consumption was inversely associated with direct measures of insulin sensitivity and clearance, these associations were mediated by dietary cholesterol or BMI in the non-diabetic multiethnic population of the IRAS.

Limited evidence is available to describe the association between egg consumption and the metabolic traits underlying type 2 diabetes. The Cardiovascular Health Study reported a significant positive association of egg consumption with fasting blood glucose and surrogate measures of insulin resistance (i.e. fasting insulin and homeostasis model assessment-insulin resistance); however, as suggested by the authors of that paper, the small absolute differences they reported were unlikely of any clinical significance ${ }^{(7)}$. No previous studies have examined these associations using detailed measures of insulin sensitivity, secretion and clearance.

Table 3 Estimated regression coefficients (and 95\% confidence intervals) on the association between egg consumption and insulin metabolism and blood glucose among non-diabetic participants ( $n$ 949) in the Insulin Resistance Atherosclerosis Study (IRAS)

\begin{tabular}{|c|c|c|c|c|c|c|c|}
\hline & \multicolumn{7}{|c|}{ Egg consumption } \\
\hline & \multicolumn{2}{|c|}{1 to $<3 /$ week $v .<1 /$ week } & \multicolumn{2}{|c|}{3 to $<5 /$ week $v .<1 /$ week } & \multicolumn{2}{|c|}{$\geq 5 /$ week $v .<1 /$ week } & \multirow[b]{2}{*}{$P$ for linear trenc } \\
\hline & $\beta$ & $95 \% \mathrm{Cl}$ & $\beta$ & $95 \% \mathrm{Cl}$ & $\beta$ & $95 \% \mathrm{Cl}$ & \\
\hline \multicolumn{8}{|l|}{ Log $S_{1}$} \\
\hline Unadjusted & -0.04 & $-0.12,0.03$ & $-0 \cdot 10$ & $-0.22,0.02$ & -0.30 & $-0.46,-0.14$ & $<0.001$ \\
\hline Model 1 & -0.04 & $-0.12,0.03$ & -0.08 & $-0.21,0.04$ & -0.25 & $-0.41,-0.10$ & 0.003 \\
\hline Model 2 & -0.05 & $-0.12,0.03$ & -0.07 & $-0.20,0.05$ & -0.27 & $-0.43,-0.11$ & 0.002 \\
\hline Model 3 & -0.03 & $-0.11,0.05$ & -0.03 & $-0 \cdot 16,0 \cdot 10$ & -0.22 & $-0.38,-0.05$ & 0.05 \\
\hline \multicolumn{8}{|c|}{ Log fasting insulin } \\
\hline Unadjusted & 0.09 & $0 \cdot 00,0 \cdot 18$ & $0 \cdot 16$ & $0.02,0.30$ & 0.45 & $0.27,0.63$ & $<0.001$ \\
\hline Model 1 & 0.09 & $0.01,0.18$ & $0 \cdot 13$ & $-0.01,0.28$ & 0.40 & $0.21,0.58$ & $<0.001$ \\
\hline Model 2 & 0.09 & $0 \cdot 00,0.18$ & $0 \cdot 12$ & $-0.02,0.27$ & 0.42 & $0.24,0.61$ & $<0.001$ \\
\hline Model 3 & 0.07 & $-0.02,0.16$ & 0.08 & $-0.07,0.23$ & 0.35 & $0.15,0.54$ & 0.002 \\
\hline \multicolumn{8}{|l|}{ Log MCRI } \\
\hline Unadjusted & -0.06 & $-0.12,0.01$ & $-0 \cdot 14$ & $-0.24,-0.03$ & -0.24 & $-0.37,-0.11$ & $<0.001$ \\
\hline Model 1 & -0.07 & $-0.13,0.00$ & -0.13 & $-0.23,-0.02$ & -0.21 & $-0.35,-0.08$ & $<0.001$ \\
\hline Model 2 & -0.07 & $-0.14,-0.01$ & $-0 \cdot 12$ & $-0.23,-0.02$ & -0.24 & $-0.38,-0.11$ & $<0.001$ \\
\hline Model 3 & -0.05 & $-0.12,0.01$ & -0.09 & $-0.20,0.02$ & -0.20 & $-0.34,-0.06$ & 0.005 \\
\hline \multicolumn{8}{|c|}{ Log $S_{1}$-adjusted AIR } \\
\hline Unadjusted & 0.00 & $-0 \cdot 10,0 \cdot 11$ & $0 \cdot 13$ & $-0.04,0.30$ & 0.11 & $-0.11,0.34$ & 0.2 \\
\hline Model 1 & 0.00 & $-0.11,0.10$ & 0.07 & $-0.11,0.24$ & 0.02 & $-0.20,0.24$ & $0 . \overline{6}$ \\
\hline Model 2 & 0.00 & $-0 \cdot 10,0 \cdot 11$ & 0.07 & $-0.11,0.24$ & -0.07 & $-0.23,0.22$ & 0.7 \\
\hline Model 3 & 0.00 & $-0.11,0.11$ & 0.06 & $-0.12,0.24$ & -0.03 & $-0.27,0.20$ & 0.9 \\
\hline \multicolumn{8}{|c|}{ Fasting blood glucose } \\
\hline Unadjusted & 0.44 & $-1 \cdot 14,2 \cdot 02$ & 0.77 & $-1 \cdot 83,3 \cdot 36$ & $2 \cdot 13$ & $-1 \cdot 18,5 \cdot 43$ & 0.2 \\
\hline Model 1 & 0.79 & $-0 \cdot 78,2 \cdot 36$ & $1 \cdot 26$ & $-1 \cdot 30,3 \cdot 83$ & $2 \cdot 62$ & $-0.67,5.92$ & 0.08 \\
\hline Model 2 & 0.68 & $-0 \cdot 88,2 \cdot 24$ & 0.85 & $-1 \cdot 72,3 \cdot 41$ & $2 \cdot 70$ & $-0.60,5.99$ & $0 \cdot 1$ \\
\hline Model 3 & 0.50 & $-1 \cdot 11,2 \cdot 12$ & 0.55 & $-2 \cdot 16,3 \cdot 25$ & $2 \cdot 10$ & $-1 \cdot 40,5 \cdot 60$ & 0.3 \\
\hline \multicolumn{8}{|c|}{ 2-h Post-load blood glucose } \\
\hline Unadjusted & $1 \cdot 26$ & $-3 \cdot 47,5 \cdot 98$ & $3 \cdot 17$ & $-4 \cdot 60,10 \cdot 94$ & $14 \cdot 37$ & $4 \cdot 47,24 \cdot 28$ & 0.02 \\
\hline Model 1 & 1.59 & $-3 \cdot 18,6 \cdot 36$ & 4.98 & $-2 \cdot 83,12 \cdot 80$ & $15 \cdot 92$ & $5 \cdot 90,25 \cdot 93$ & 0.005 \\
\hline Model 2 & $1 \cdot 75$ & $-2.99,6.48$ & 3.66 & $-4 \cdot 12,11 \cdot 44$ & $16 \cdot 46$ & $6 \cdot 48,26 \cdot 45$ & 0.006 \\
\hline Model 3 & $1 \cdot 31$ & $-3.57,6 \cdot 20$ & $3 \cdot 12$ & $-5 \cdot 05,11 \cdot 29$ & $13 \cdot 74$ & $3 \cdot 16,24 \cdot 32$ & 0.04 \\
\hline
\end{tabular}

$\mathrm{S}_{\mathrm{I}}$, insulin sensitivity; MCRI, metabolic clearance of insulin; AIR, acute insulin response.

Model 1: adjusted for age, sex, ethnicity, study centre.

Model 2: Model 1 + education, income, smoking, alcohol consumption, energy expenditure, family history of diabetes.

Model 3: Model 2 + dietary factors (processed meats, fast foods, red meats, dairy products, fruits, whole grains, coffee and tea).

Unadjusted and multivariable-adjusted linear regression analyses were used to explore the association between egg consumption and each outcome measure. 
Table 4 Estimated regression coefficients (and 95\% confidence intervals) on the association between egg consumption and insulin metabolism and blood glucose, adjusted for potential mediators, among non-diabetic participants ( $n 937$ to 942 ) in the Insulin Resistance Atherosclerosis Study (IRAS)

\begin{tabular}{|c|c|c|c|c|c|c|c|}
\hline & \multicolumn{7}{|c|}{ Egg consumption } \\
\hline & \multicolumn{2}{|c|}{1 to $<3 /$ week $v$. $<1 /$ week } & \multicolumn{2}{|c|}{3 to $<5 /$ week $v$. $<1 /$ week } & \multicolumn{2}{|c|}{$\geq 5$ /week v. <1/week } & \multirow[b]{2}{*}{$P$ for linear trend } \\
\hline & $\beta$ & $95 \% \mathrm{Cl}$ & $\beta$ & $95 \% \mathrm{Cl}$ & $\beta$ & $95 \% \mathrm{Cl}$ & \\
\hline \multicolumn{8}{|l|}{$\log S_{1}$} \\
\hline + energy & -0.02 & $-0 \cdot 10,0.06$ & -0.01 & $-0 \cdot 14,0.12$ & $-0 \cdot 19$ & $-0.36,-0.03$ & 0.01 \\
\hline+ saturated fat & -0.02 & $-0 \cdot 10,0.05$ & -0.03 & $-0 \cdot 16,0 \cdot 10$ & -0.22 & $-0.39,-0.05$ & 0.05 \\
\hline + cholesterol & -0.04 & $-0.13,0.05$ & -0.08 & $-0 \cdot 26,0 \cdot 10$ & -0.31 & $-0.61,-0.01$ & $0 \cdot 2$ \\
\hline$+\mathrm{BMI}$ & -0.09 & $-0.07,0.07$ & 0.02 & $-0 \cdot 10,0 \cdot 14$ & -0.09 & $-0.24,0.06$ & 0.6 \\
\hline+ WHR & -0.04 & $-0.11,0.03$ & -0.02 & $-0 \cdot 14,0 \cdot 10$ & $-0 \cdot 16$ & $-0.31,-0.00$ & 0.04 \\
\hline \multicolumn{8}{|l|}{ Log fasting insulin } \\
\hline + energy & 0.07 & $-0.03,0.17$ & 0.05 & $-0 \cdot 10,0 \cdot 21$ & $0 \cdot 32$ & $0 \cdot 13,0.52$ & 0.007 \\
\hline+ saturated fat & 0.07 & $-0.03,0.16$ & 0.06 & $-0.09,0.21$ & $0 \cdot 33$ & $0.13,0.53$ & 0.005 \\
\hline + cholesterol & 0.06 & $-0 \cdot 05,0 \cdot 16$ & 0.03 & $-0 \cdot 17,0 \cdot 24$ & $0 \cdot 26$ & $-0.09,0.60$ & $0 \cdot 3$ \\
\hline + BMI & 0.04 & $-0.04,0.12$ & 0.00 & $-0 \cdot 14,0 \cdot 13$ & $0 \cdot 21$ & $0.04,0.38$ & 0.08 \\
\hline+ WHR & 0.08 & $0 \cdot 00,0 \cdot 16$ & 0.05 & $-0.09,0.19$ & 0.29 & $0.11,0.47$ & 0.005 \\
\hline \multicolumn{8}{|l|}{ Log MCRI } \\
\hline + energy & -0.04 & $-0.11,0.02$ & -0.07 & $-0.18,0.04$ & $-0 \cdot 17$ & $-0.31,-0.03$ & 0.02 \\
\hline+ saturated fat & -0.04 & $-0.11,0.02$ & -0.07 & $-0 \cdot 18,0.04$ & $-0 \cdot 18$ & $-0.32,-0.04$ & 0.02 \\
\hline + cholesterol & -0.04 & $-0.12,0.03$ & -0.06 & $-0.21,0.09$ & $-0 \cdot 15$ & $-0 \cdot 40,0 \cdot 11$ & $0 \cdot 3$ \\
\hline + BMI & -0.03 & $-0.08,0.03$ & -0.03 & $-0 \cdot 12,0 \cdot 07$ & -0.08 & $-0.21,0.04$ & 0.2 \\
\hline+ WHR & -0.06 & $-0 \cdot 12,0.00$ & -0.07 & $0 \cdot 17,0.03$ & $-0 \cdot 15$ & $0.28,-0.02$ & 0.01 \\
\hline \multicolumn{8}{|l|}{ Log $S_{1}$-adjusted AIR } \\
\hline + energy & 0.00 & $-0 \cdot 11,0 \cdot 11$ & 0.06 & $-0 \cdot 13,0 \cdot 24$ & -0.04 & $-0 \cdot 27,0 \cdot 20$ & 0.9 \\
\hline+ saturated fat & 0.00 & $-0 \cdot 11,0 \cdot 11$ & 0.05 & $-0 \cdot 14,0 \cdot 23$ & -0.05 & $-0 \cdot 29,0 \cdot 19$ & $1 \cdot 0$ \\
\hline + cholesterol & 0.03 & $-0 \cdot 10,0 \cdot 15$ & $0 \cdot 13$ & $-0 \cdot 12,0 \cdot 38$ & $0 \cdot 11$ & $-0.31,0.53$ & $0 \cdot 4$ \\
\hline$+\mathrm{BMI}$ & 0.00 & $-0 \cdot 11,0 \cdot 10$ & 0.06 & $-0.12,0.24$ & -0.04 & $-0.28,0.19$ & $1 \cdot 0$ \\
\hline + WHR & 0.00 & $-0 \cdot 10,0 \cdot 11$ & 0.07 & $-0 \cdot 12,0.25$ & -0.03 & $-0.27,0.20$ & $0 \cdot 9$ \\
\hline \multicolumn{8}{|c|}{ Fasting blood glucose } \\
\hline + energy & 0.35 & $-1 \cdot 26,1 \cdot 97$ & 0.25 & $-2 \cdot 47,2 \cdot 97$ & $1 \cdot 75$ & $-1 \cdot 77,5 \cdot 26$ & 0.4 \\
\hline+ saturated fat & $0 \cdot 40$ & $-1 \cdot 22,2 \cdot 03$ & 0.46 & $-2 \cdot 29,3 \cdot 20$ & $2 \cdot 00$ & $-1 \cdot 54,5 \cdot 54$ & $0 \cdot 3$ \\
\hline + cholesterol & 0.02 & $-1 \cdot 83,1 \cdot 86$ & -0.65 & $-4 \cdot 36,3 \cdot 06$ & $-0 \cdot 27$ & $-6 \cdot 49,5 \cdot 96$ & $0 \cdot 9$ \\
\hline$+\mathrm{BMI}$ & $0 \cdot 17$ & $-1 \cdot 36,1 \cdot 71$ & -0.48 & $-3 \cdot 06,2 \cdot 11$ & 0.37 & $-2 \cdot 96,3 \cdot 71$ & $1 \cdot 0$ \\
\hline+ WHR & 0.58 & $-1 \cdot 00,2 \cdot 15$ & $0 \cdot 21$ & $-2 \cdot 44,2 \cdot 86$ & $1 \cdot 39$ & $-2 \cdot 03,4 \cdot 80$ & 0.4 \\
\hline \multicolumn{8}{|c|}{ 2-h Post-load blood glucose } \\
\hline + energy & $1 \cdot 21$ & $-3 \cdot 70,6 \cdot 11$ & $2 \cdot 64$ & $-5 \cdot 60,10 \cdot 88$ & $13 \cdot 19$ & $2 \cdot 53,23 \cdot 85$ & 0.05 \\
\hline+ saturated fat & $1 \cdot 67$ & $-3 \cdot 25,6 \cdot 59$ & $4 \cdot 18$ & $-4 \cdot 12,12 \cdot 49$ & 14.99 & $4 \cdot 27,25 \cdot 70$ & 0.02 \\
\hline + cholesterol & 0.96 & $-4 \cdot 63,6 \cdot 55$ & $2 \cdot 11$ & $-9 \cdot 13,13 \cdot 35$ & $11 \cdot 64$ & $-7 \cdot 21,30 \cdot 50$ & $0 \cdot 6$ \\
\hline+ BMI & 0.09 & $-4 \cdot 57,4 \cdot 74$ & $0 \cdot 48$ & $-7 \cdot 37,8 \cdot 32$ & $8 \cdot 77$ & $-1 \cdot 35,18 \cdot 89$ & $0 \cdot 3$ \\
\hline+ WHR & $1 \cdot 62$ & $-3 \cdot 03,6 \cdot 27$ & $2 \cdot 05$ & $-5 \cdot 77,9 \cdot 87$ & $10 \cdot 91$ & $0 \cdot 83,20 \cdot 99$ & 0.03 \\
\hline
\end{tabular}

$\mathrm{S}_{\mathrm{I}}$ insulin sensitivity; HR, waist:hip ratio; MCRI, metabolic clearance of insulin; AIR, acute insulin response.

Base model adjusted age, sex, ethnicity, study centre, education, income, smoking, alcohol consumption, energy expenditure, family history of diabetes and dietary factors; each row represents the addition to this model of the individual variable indicated.

Unadjusted and multivariable-adjusted linear regression analyses were used to determine the association between egg consumption and each outcome measure.

Table 5 Sobel-Goodman mediation analysis for the association of egg consumption with insulin sensitivity and clearance among non-diabetic participants ( $n 937$ to 942) in the Insulin Resistance Atherosclerosis Study (IRAS)

\begin{tabular}{llcr}
\hline Potential mediator & Outcome & Proportion of total effect that is mediated (\%) & $P$ value \\
\hline Dietary cholesterol & S $_{\text {I }}$ & 47 & 0.02 \\
& Fasting insulin & 75 & 0.004 \\
MCRI & 61 & $<0.001$ \\
BMI & S $_{\text {I }}$ & 54 & $<0.001$ \\
& Fasting insulin & 42 & $<0.001$ \\
& MCRI & 53 & $<0.001$
\end{tabular}

$\mathrm{S}_{\mathrm{I}}$, insulin sensitivity; MCRI, metabolic clearance of insulin.

Sobel-Goodman mediation tests were used to determine whether dietary cholesterol or BMI mediates the association of egg consumption with insulin sensitivity and clearance.

Our findings suggest a strong mediating role of dietary cholesterol in the association of egg consumption with insulin sensitivity and clearance. Dietary cholesterol has been shown to be associated with an increased risk of incident type 2 diabetes $^{(3,18)}$ and gestational diabetes ${ }^{(6)}$. Egg consumption has traditionally been accompanied by food choices that are high in fat and cholesterol, excessive dietary cholesterol could result in the accumulation 
of hepatic cholesterol esters and TAG, which in turn activate liver $\mathrm{X}$ receptors (LXR), nuclear receptors that regulate hepatic lipid metabolism ${ }^{(19)}$. LXR activation decreases insulin receptor $\beta$-subunit mass, reduces insulin receptor substrate-1 tyrosine phosphorylation and increases protein tyrosine phosphatase IB protein mass; all of these metabolic derangements interrupt hepatic insulin signalling and aggravate insulin resistance ${ }^{(20)}$. Experimental evidence has shown that treating insulin-resistant mice with synthetic LXR ligands improves glucose tolerance and increases peripheral glucose uptake ${ }^{(21)}$.

A number of previous prospective studies have shown an independent association between egg consumption and incident diabetes ${ }^{(3-6)}$. While these studies have adjusted for a wide range of potential confounders in the regression analyses, only one of them has accounted for dietary cholesterol ${ }^{(3)}$. In the Women's Health Study, the association between egg consumption and type 2 diabetes was attenuated after additional adjustment for dietary cholesterol $^{(3)}$.

BMI also mediates the association of egg consumption with insulin sensitivity and clearance. As discussed earlier, egg consumption is often accompanied by food choices that are high in fat and cholesterol in Western countries; thus, it is plausible that excessive intakes of these high-fat and energy-dense foods increase the risk of diabetes through obesity which leads to insulin resistance, inflammation and other disorders in the pathogenesis of diabetes. We have previously reported in the IRAS cohort that higher egg consumption was clustered in a dietary pattern with red meat, low-fibre bread and cereals, tomato vegetables, fried potatoes and cheese, which in turn increased the risk of incident diabetes ${ }^{(22)}$. To support this notion, a recent study demonstrated that intake of restaurant meals was associated with risk of incident diabetes and this association was mediated by weight gain and obesity ${ }^{(23)}$. In addition, further adjustment for the consumption of processed meats and fast foods attenuated the associations of egg consumption with insulin sensitivity and clearance, which provides further support that these associations were mediated by food choices which are characterized by high dietary intake of cholesterol. Lastly, egg consumption may cluster with other lifestyle factors that impact on insulin sensitivity and clearance. We have adjusted for alcohol consumption, smoking, physical activity and a wide range of food items that were correlated with egg consumption in IRAS. However, we could not rule out the possibility of residual confounding by factors that we did not measure or measured imprecisely.

The strengths of the present study included the wellcharacterized multi-ethnic population of the IRAS cohort and the detailed measurements of insulin sensitivity, secretion and clearance. Our study has potential limitations. Egg consumption was measured by self-report which increases the chance of measurement error and bias.
If the measurement errors were random, then the inverse association of egg consumption with insulin sensitivity and clearance would be stronger than we observed. However, any systematic difference in reporting egg consumption based on obesity status or social desirability would bias the association in either direction. We did not have information on cooking methods. Individuals who habitually consume poached or boiled eggs may be characterized by different lifestyle patterns which may modify their risk of diabetes. Finally, our results may not be generalizable to populations with other ethnic backgrounds.

\section{Conclusion}

The associations that we observed suggest that egg consumption is associated with lower insulin sensitivity and clearance, but that these associations are mediated by dietary cholesterol or BMI. Our findings reinforce public health recommendations to limit dietary intake of cholesterol and to reduce weight as preventive strategies for type 2 diabetes.

\section{Acknowledgements}

Sources of funding: C.C.L. is supported by a postdoctoral research fellowship from the Banting \& Best Diabetes Centre, University of Toronto, Canada. A.J.H. holds a Tier II Canada Research Chair in Diabetes Epidemiology. IRAS was supported by grants U01-HL47892, U01-HL47902, DK-29867 and R01-58329 from the National Heart, Lung, and Blood Institute, and by grant M01-RR-43 from the National Institutes of Health. Conflicts of interest: The authors declare that there is no conflict of interest associated with this manuscript. Authors' contributions: L.E.W., S.M.H. and M.J.R. designed the study. C.C.L. and A.J.H. analysed the data and wrote the manuscript. All authors contributed to the discussion and approved the contents of the manuscript.

\section{References}

1. Hu FB, Stampfer MJ, Rimm EB et al. (1999) A prospective study of egg consumption and risk of cardiovascular disease in men and women. JAMA 282, 1387-1394.

2. Djoussé L \& Gaziano JM (2008) Egg consumption in relation to cardiovascular disease and mortality: the Physicians' Health Study. Am J Clin Nutr 87, 964-969.

3. Djoussé L, Gaziano JM, Buring JE et al. (2009) Egg consumption and risk of type 2 diabetes in men and women. Diabetes Care 32, 295-300.

4. Shi Z, Yuan B, Zhang C et al. (2011) Egg consumption and the risk of diabetes in adults, Jiangsu, China. Nutrition $\mathbf{2 7}$, 194-198.

5. Radzevičiené L \& Ostrauskas R (2012) Egg consumption and the risk of type 2 diabetes mellitus: a case-control study. Public Health Nutr 15, 1437-1441. 
6. Qiu C, Frederick IO, Zhang C et al. (2011) Risk of gestational diabetes mellitus in relation to maternal egg and cholesterol intake. Am J Epidemiol 175, 649-658.

7. Djoussé L, Kamineni A, Nelson TL et al. (2010) Egg consumption and risk of type 2 diabetes in older adults. Am J Clin Nutr 92, 422-427.

8. Wagenknecht LE, Mayer EJ, Rewers M et al. (1995) The insulin resistance atherosclerosis study (IRAS) objectives, design, and recruitment results. Ann Epidemiol 5, 464-472.

9. Mayer-Davis EJ, Vitolins MZ, Carmichael SL et al. (1999) Validity and reproducibility of a food frequency interview in a multi-cultural epidemiologic study. Ann Epidemiol 9, 314-324.

10. Bergman RN, Finegood DT \& Ader M (1985) Assessment of insulin sensitivity in vivo. Endocr Rev 16, 45-86.

11. Welch S, Gebhart SSP, Bergman RN et al. (1990) Minimal model analysis of intravenous glucose tolerance test derived insulin sensitivity in diabetic subjects. J Clin Endocrinol Metab 71, 1508-1518.

12. Steil GM, Volund A, Kahn SE et al. (1993) Reduced sample number for calculation of insulin sensitivity and glucose effectiveness from the minimal model: suitability for use in population studies. Diabetes 42, 250-256.

13. Pacini G \& Bergman RN (1986) MINMOD: a computer program to calculate insulin sensitivity and pancreatic responsivity from the frequently sampled intravenous glucose tolerance test. Comput Methods Programs Biomed 23, 113-122.

14. Lorenzo C, Wagenknecht LE, D'Agostino RB Jr et al. (2010) Insulin resistance, $\beta$-cell dysfunction, and conversion to type 2 diabetes in multiethnic population: the Insulin Resistance Atherosclerosis Study. Diabetes Care 33, 67-72.
15. Polonsky KS, Pugh W, Jaspan JB et al. (1984) Relationship between peripheral concentrations of C-peptide and insulin and their secretion rates in the dog. J Clin Invest 74, 1821-1829.

16. Howard BV, Mayer-Davis EJ, Goff D et al. (1998) Relationships between insulin resistance and lipoproteins in nondiabetic African Americans, Hispanics, and non-Hispanic whites: the Insulin Resistance Atherosclerosis Study. Metabolism 47, 1174-1179.

17. Mackinnon DP, Warsi G \& Dwyer JH (1995) A simulation study of mediated effect measures. Multivariate Behav Res 30, 41-62.

18. Meyer KA, Kushi LH \& Jacob DR Jr et al. (2001) Dietary fat and incidence of type 2 diabetes in older Iowa women. Diabetes Care 24, 1528-1535.

19. Basciano H, Miller AE, Naples M et al. (2009) Metabolic effects of dietary cholesterol in an animal model of insulin resistance and hepatic steatosis. Am J Physiol Endocrinol Metab 297, E462-E473.

20. Basciano H, Miller AE, Baker C et al. (2009) LXR $\alpha$ activation perturbs hepatic insulin signaling and stimulates production of apolipoprotein B-containing lipoproteins. $\mathrm{Am} \mathrm{J}$ Physiol Gastrointest Liver Physiol 297, G323-G332.

21. Commerford SR, Vargas L, Dorfman SE et al. (2007) Dissection of the insulin-sensitizing effect of liver $\mathrm{X}$ receptor ligands. Mol Endocrinol 21, 3002-3012.

22. Liese AD, Weis KE, Schulz M et al. (2009) Food intake patterns associated with incident type 2 diabetes: the Insulin Resistance Atherosclerosis Study. Diabetes Care 32, 263-268.

23. Krishnan S, Coogan PF, Boggs DA et al. (2010) Consumption of restaurant foods and incidence of type 2 diabetes in African-American women. Am J Clin Nutr 91, 465-471. 